\title{
AuNP-Amplified Surface Acoustic Wave Sensor for the Quantification of Exosomes
}

Chenyun Wang", Cencen Wang", Dan Jin, Yi Yu, Fan Yang, Yulin Zhang, Qunfeng Yao, Guo-Jun Zhang*

School of Laboratory Medicine, Hubei University of Chinese Medicine, 16 Huangjia Lake West Road, Wuhan 430065, P.R. China

\# These authors contributed equally to this work.

*Corresponding author: Tel: +86-27-68890259, Fax: +86-27-68890259

E-mail: zhanggj@hbtcm.edu.cn 


\section{Characterization of Exosomes}

\section{TEM}

Exosomes were analyzed by negative staining transmission electron microscopy (TEM, Hitachi H-7000 NAR) as previously described. 400-mesh carbon-coated copper grid were floated (carbon side down) on top of $20 \mu \mathrm{L}$ of exosome suspensions for $5 \mathrm{~min}$. The samples were then fixed on the copper grid for $20 \mathrm{~min}$ with $2 \%$ glutaraldehyde, followed by dried in the room temperature. Grids were then dipped in $2 \%$ phosphotungstic acid for dyeing purpose. Images were obtained by the TEM operating at $80 \mathrm{kV}$ soon after the samples were rinsed with PBS and dried at room temperature.

\section{NTA}

Nanoparticle tracking analysis (NTA) was used to analysis the size distribution and concentration of exosome particles. Briefly, suspensions were diluted in PBS till an optimum concentration of particles was obtained (1/400) after filtrating through a $0.22-\mu \mathrm{m}$ pore filter. After that, samples were injected to the equipment and illuminated by NTA laser beam. The typical brownian motion of exosomes could scatter lights, and be observed by a microscope equipped with a $405 \mathrm{~nm}$ laser and sCMOS camera (Zeta view Ltd., Germany). With the optimal settings, the individual exosomes in the suspensions could be visualized. All tests were performed at room temperature. Each sample was tested 3 times.

\section{Western blot:}

Exosomes were diluted in PBS and lysed using a radio-immunoprecipitation buffer containing protease inhibitors (Servicebio, Wuhan, China). In this work, the protein was quantified using the manufacturer's recommended bicinchoninic acid assay. The protein was then separated using gel electrophoresis and transferred to the polyvinylidene fluoride membrane. Blots were further inmmunoblotted with primary antibodies against CD63 and EpCAM (Abcam, 1:500 solution) overnight at $4^{\circ} \mathrm{C}$. Subsequently, the blots were incubated with HRP-conjugated secondary antibody (1:3000 solution) at room temperature for $1 \mathrm{~h}$. Finally, a chemiluminescence system (Bio Rad, U.S.A) was used to visualize the immunoreactive bands. 


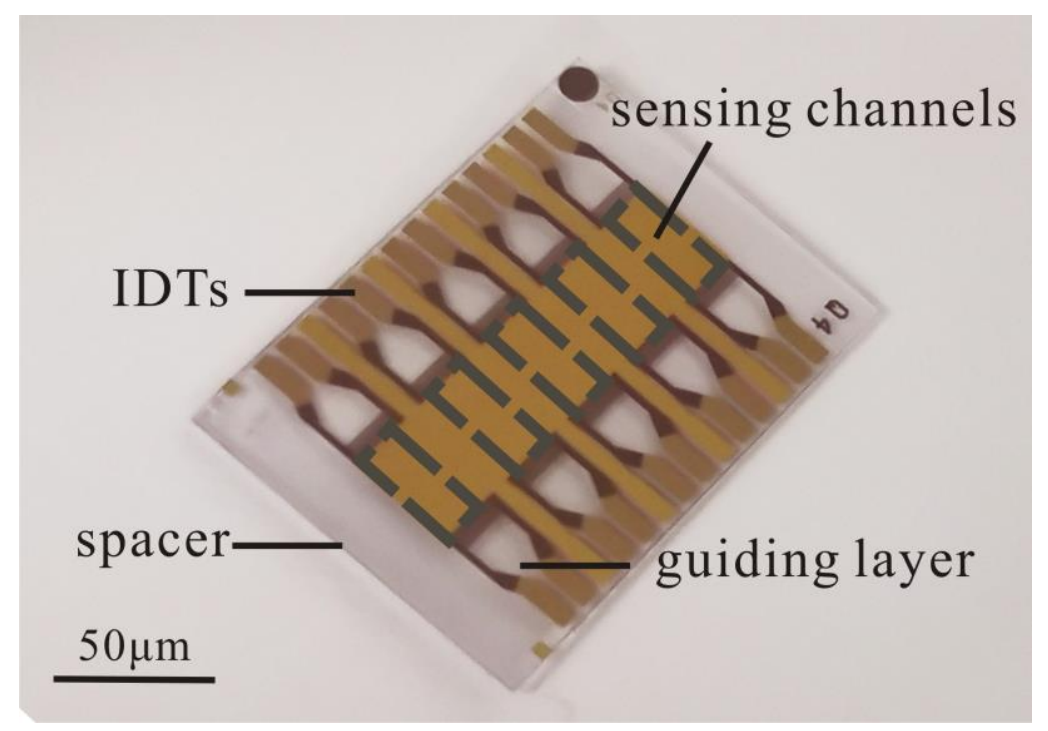

Figure S1. The optical image of the SAW chip layout.

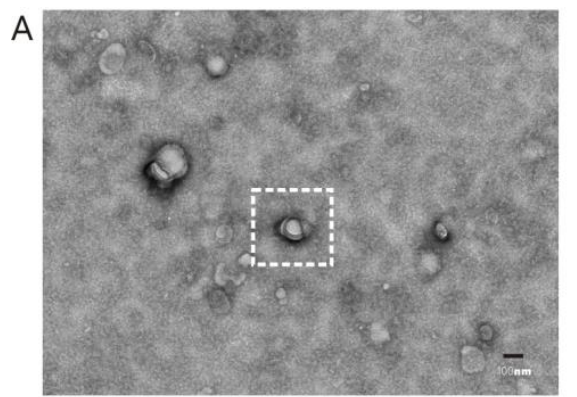

C

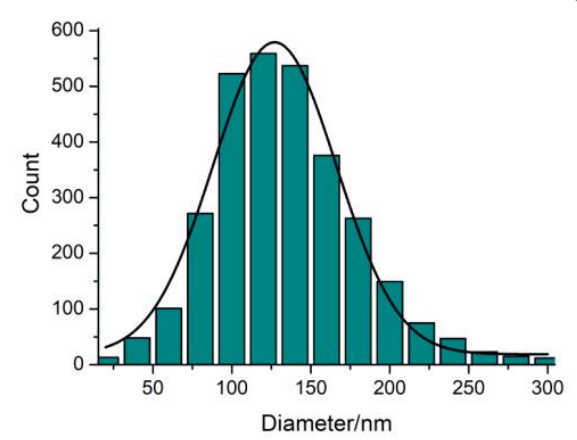

B

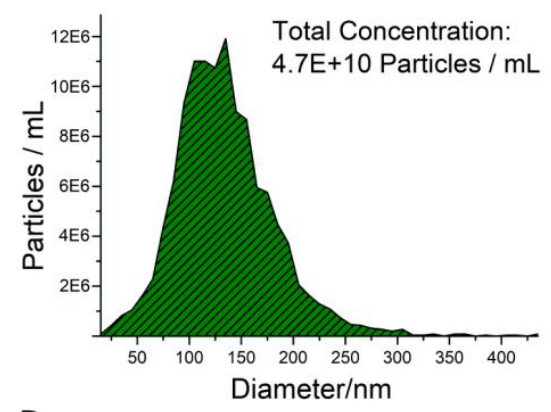

D
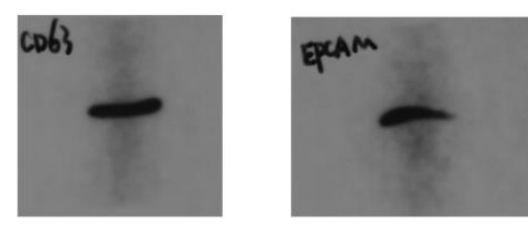

Figure S2. The characterization of exosomes. (A) TEM image of the purified exosome derived by HepG2 cells. It shows a typical cup-shaped structure of exosome. (B) The concentration of HepG2 exosomes obtained by NTA. (C) Size distribution of HepG2 exosomes obtained by NTA. (D) Detection of exosomal protein markers (CD63 and EpCAM) from HepG2 exosomes by western blotting. 


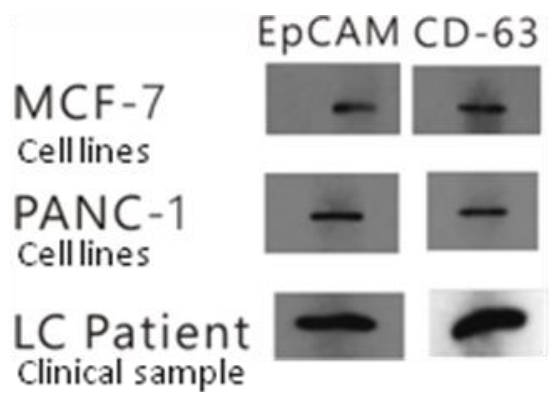

Figure S3. The western blot results of exosomes from MCF-7, PANC-1 cell lines and blood sample from lung cancer patients, respectively.

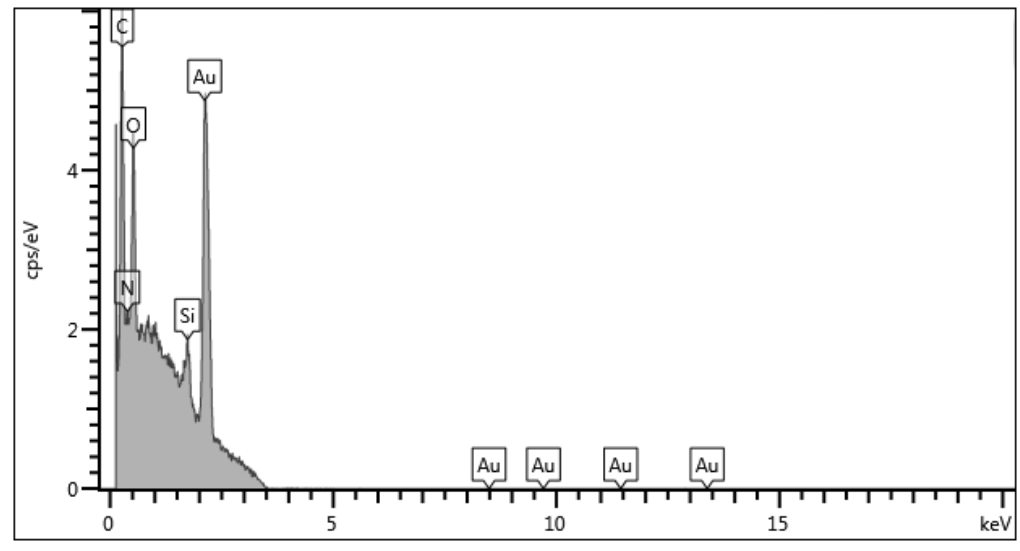

Figure S4. The EDS characterization of the SAW chips. The elemental analysis confirms the presence of the AuNP. 

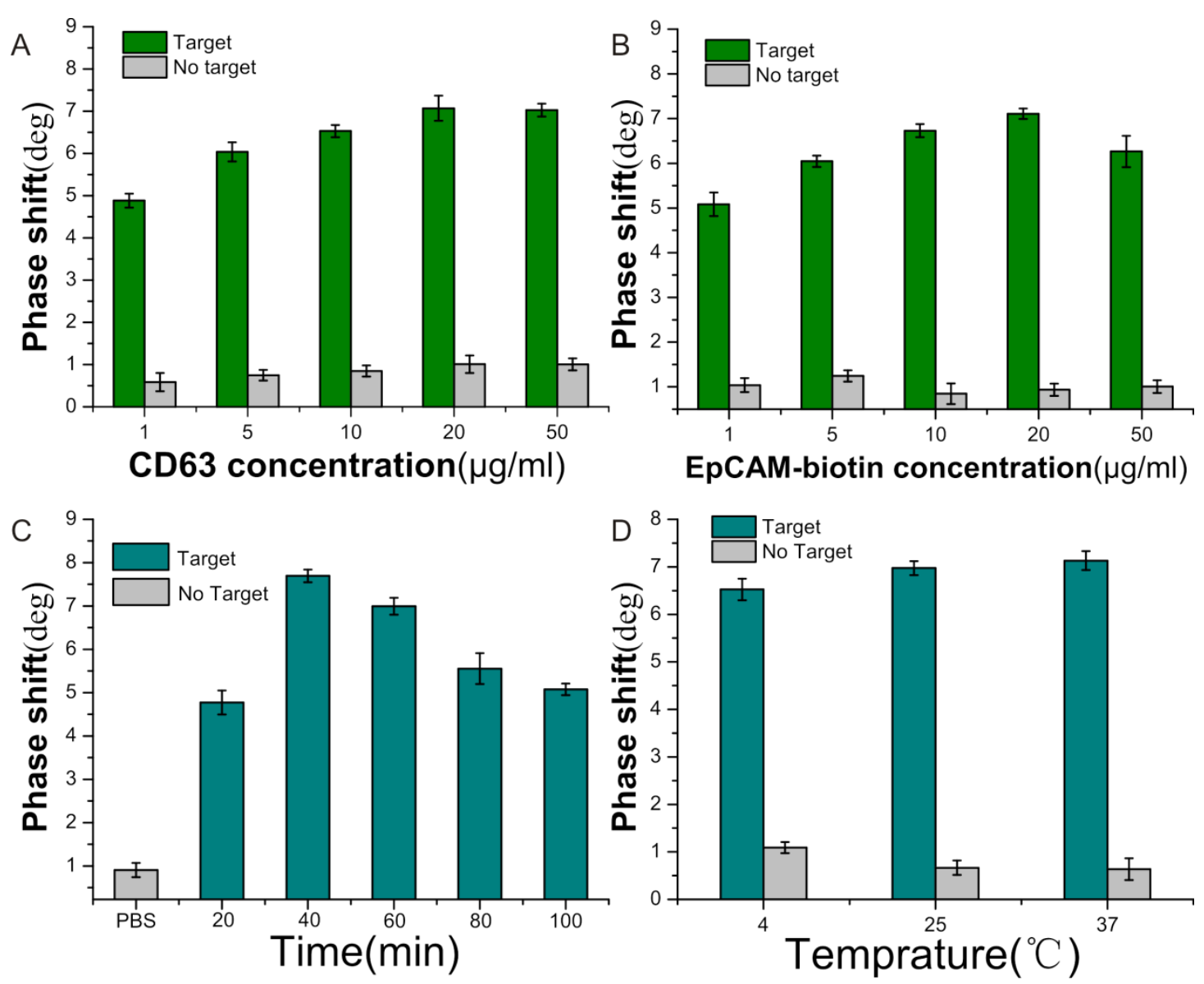

Figure S5. The influence of different parameters on the SAW sensor performance. (A) The phase shift versus different anti-CD63 antibody concentrations $(20 \mu \mathrm{g} / \mathrm{mL}$ as the biotin-modified EpCAM antibody concentration, $37^{\circ} \mathrm{C}$ as the reaction temperature, 40 min as the incubation time). (B) The phase shift versus different biotin modified anti-EpCAM antibody concentrations $(20 \mu \mathrm{g} / \mathrm{mL}$ as the CD63 antibody concentration, $37^{\circ} \mathrm{C}$ as the reaction temperature, $40 \mathrm{~min}$ as the incubation time). (C) The phase shift versus different incubation time $(20 \mu \mathrm{g} / \mathrm{mL}$ as the CD63 antibody concentration, 20 $\mu \mathrm{g} / \mathrm{mL}$ as the biotin-modified EpCAM antibody concentration, $37^{\circ} \mathrm{C}$ as the reaction temperature). (D) The phase shift versus different temperature $(20 \mu \mathrm{g} / \mathrm{mL}$ as the CD63 antibody concentration, $20 \mu \mathrm{g} / \mathrm{mL}$ as the biotin-modified EpCAM antibody concentration, $40 \mathrm{~min}$ as the incubation time). Error bars represent standard deviations of measurements $(n=3)$. 
Figure S6: The TEM image of the purified MV. 
Table S1. The basic patient and healthy control information

\begin{tabular}{|c|c|c|c|c|c|c|}
\hline Number & $\begin{array}{l}\text { patient's } \\
\text { condition }\end{array}$ & $\begin{array}{c}\text { patient's } \\
\text { age }\end{array}$ & $\begin{array}{c}\text { patient's } \\
\text { sex }\end{array}$ & $\begin{array}{c}\text { sample } \\
\text { status }\end{array}$ & anticoagulant & volume \\
\hline 1 & $\mathrm{LC}$ & 54 & $\mathrm{~F}$ & Frozen & Negative & $500 \mu \mathrm{L}$ \\
\hline 2 & $\mathrm{LC}$ & 39 & M & Frozen & Negative & $500 \mu \mathrm{L}$ \\
\hline 3 & $\mathrm{LC}$ & 70 & M & Frozen & Negative & $500 \mu \mathrm{L}$ \\
\hline 4 & $\mathrm{LC}$ & 41 & M & Frozen & Negative & $500 \mu \mathrm{L}$ \\
\hline 5 & $\mathrm{LC}$ & 52 & M & Frozen & Negative & $500 \mu \mathrm{L}$ \\
\hline 6 & $\mathrm{HCC}$ & 37 & $\mathrm{~F}$ & Frozen & Negative & $500 \mu \mathrm{L}$ \\
\hline 7 & $\mathrm{HCC}$ & 44 & $\mathrm{~F}$ & Frozen & Negative & $500 \mu \mathrm{L}$ \\
\hline 8 & $\mathrm{HCC}$ & 50 & M & Frozen & Negative & $500 \mu \mathrm{L}$ \\
\hline 9 & $\mathrm{HCC}$ & 69 & M & Frozen & Negative & $500 \mu \mathrm{L}$ \\
\hline 10 & $\mathrm{HCC}$ & 47 & $\mathrm{~F}$ & Frozen & Negative & $500 \mu \mathrm{L}$ \\
\hline 11 & Healthy & 28 & $\mathrm{~F}$ & Frozen & Negative & $500 \mu \mathrm{L}$ \\
\hline 12 & Healthy & 33 & $\mathrm{~F}$ & Frozen & Negative & $500 \mu \mathrm{L}$ \\
\hline 13 & Healthy & 48 & M & Frozen & Negative & $500 \mu \mathrm{L}$ \\
\hline 14 & Healthy & 56 & $\mathrm{~F}$ & Frozen & Negative & $500 \mu \mathrm{L}$ \\
\hline 15 & Healthy & 53 & $\mathrm{~F}$ & Frozen & Negative & $500 \mu \mathrm{L}$ \\
\hline 16 & Healthy & 34 & M & Frozen & Negative & $500 \mu \mathrm{L}$ \\
\hline 17 & Healthy & 67 & M & Frozen & Negative & $500 \mu \mathrm{L}$ \\
\hline 18 & Healthy & 59 & M & Frozen & Negative & $500 \mu \mathrm{L}$ \\
\hline 19 & Healthy & 30 & F & Frozen & Negative & $500 \mu \mathrm{L}$ \\
\hline 20 & Healthy & 43 & $\mathrm{~F}$ & Frozen & Negative & $500 \mu \mathrm{L}$ \\
\hline
\end{tabular}

